\title{
FIELD EMISSION STUDY OF GERMANIUM THIN FILMS ON A NIOBIUM SURFACE
}

\author{
R.Z. BAKHTIZIN ${ }^{a}$, A.L. SUVOROV ${ }^{b}$ AND R.F. ZARIPOV ${ }^{a}$ \\ a Department of Physical Electronics, Bashkir State University \\ 450074 Ufa, 32 Frunze Street, Russia \\ ' Institute of Theoretical and Experimental Physics \\ 117209 Moscow, 25 B. Cheremushkinskaya Street, Russia
}

(Received May 21, 1991; in revised form October 31, 1991)

The behaviour of germanium on atomically clean niobium single crystal planes has been studied by probe-hole field emission microscopy as well as by means of the spectral analysis of field emission current fluctuations. Variations of average work function, surface diffusion activation energies, and slopes of the spectral density fuuction with different coverages and substrate temperatures are measured.

PACS numbers: $78.50 . G e$

\section{Introduction}

Investigations of adsorption processes on metals are of considerable and long standing interest for surface physics due to technological and academic importance [1]; that is why essential attention is paid to information about thin films characteristics, the initial stages of the growth, stability under ion bombardment, etc. Investigation of such systems with unusual combination of materials is supposed to be expedient $[2,3]$.

The purpose of this work is to study the emission properties of germanium thin films on niobium surface, using field electron microscope of the Muller probe-hole type. Moreover spectral analysis of the low frequency field emission current fluctuations was used. The results obtained have been compared with characteristics of Ge-W system $[4,5]$. 


\section{Experimental}

Niobium field emitters used for the present study were prepared by electrochemical etching in a $1 \mathrm{HF} / 9 \mathrm{HNO}_{3}$ mixture of a $0.10 \mathrm{~mm}$ diameter $\mathrm{Nb}$ wire (purity better than $99,9 \%$ ) spotwelded to a $0.10 \mathrm{~mm}$ diameter $\mathrm{W}$ filament. The emitters were cleaned by rneans of the following procedure which was found to be reproducible and did not lead to excessive tip blunting: several cycles of heating to $1800 \mathrm{~K}$ in $10^{-8} \mathrm{~Pa}$ and subsequently flashing in ultra-high vacuum up to $2100 \mathrm{~K}$. A field emission image of a clean $\mathrm{Nb}$ tip is shown in Fig. 1. The observed pattern

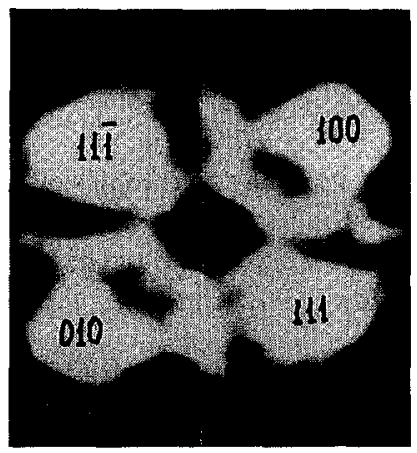

Fig. 1. Field emission pattern of a clean Nb lip.

is in agreement with former observation [6].The disposition of some crystal faces on the emitter has been indicated for the purpose of comparison.

The germanium evaporation source has been described previously $[4,5]$. The source operation was controlled by radio-frequency mass spectrometer. After thorough degassing of the evaporation source a residual gas pressure was less than $10^{-8} \mathrm{~Pa}$ during the film deposition.

Work functions $\varphi$ were determined from the variation of emission current $I$ with applied voltage $V$ and the Fowler-Nordheim equation

$$
I=A \cdot V^{2} \exp \left(-B \varphi^{3 / 2} / V\right)
$$

where $A$ is the pre-exponential term, and the constant $B$ containing the field-voltage proportionality was determined from Fowler-Nordheim plots for a clean $\mathrm{Nb}$ emitter using a value of $4.0 \mathrm{eV}$ for the average work function. Work functions were also calculated using the Klein equation [7]

$$
\varphi_{\mathrm{Ge}-\mathrm{Nb}}=\varphi_{\mathrm{Nb}}\left(V_{\mathrm{Ge}-\mathrm{Nb}} / V_{\mathrm{Nb}}\right)^{2 / 3}
$$

where $V_{\mathrm{Ge}-\mathrm{Nb}}, V_{\mathrm{Nb}}, \varphi_{\mathrm{Ge}-\mathrm{Nb}}$, and $\varphi_{\mathrm{Nb}}$ are the voltages required to draw a given total emission current $10^{-9} \mathrm{~A}$ and the work functions of the Ge-covered and clean $\mathrm{Nb}$ emitters, respectively. The applicability of this equation to determine $\varphi$ was established previously [8]. The thickness of Ge coverage was determined by the time of evaporation source operation; the estimation of coverage $\theta$ will be given further. 
All experiments were performed under oil-free ultra-high vacuum (UHV) chamber containing 3 -dimensional manipulator and air-lock system. Field electron microscope with $1.0 \mathrm{~mm}$ probe hole and field-emission energy analyser were also placed in it.

The spectral analysis of fluctuations (up to field emission current $10^{-13} \mathrm{~A}$ ) was carried out using a microcomputer system [9] in the frequency range $10^{-2}-10^{4}$ $\mathrm{Hz}$ by the fast Fourier transformation algorithm [10]. All the measurements (field emission noise spectra, current-voltage characteristics, field emission energy distributions) and observation of field emission images were taken at room temperature.

\section{Results and discussion}

\subsection{Work function of the field emitter surface}

Unlike Ge-W system germanium adsorption on niobium surface results in a decrease in the work function. Figure 2 illustrates a number of Fowler-Nordheim plots obtained for different Ge coverages at $300 \mathrm{~K}$. Ge was deposited on the total emitting surface simultaneously. Using Fowler-Nordheim data a plot of variation of average work function (over the emitter surface) with the deposition time, (i.e. with Ge coverage $\varphi(\theta)$ ) was made (Fig. 3). It is clear that with increasing coverage the work function decreased to a minimum $\varphi_{\mathrm{Ge}-\mathrm{Nb}}=3.5 \pm 0.05$ $\mathrm{eV}$ followed by an increase, levelling out at about $3.55 \mathrm{eV}$. This behaviour was found to be reproducible for a large number of experimental runs and 10 different emitter tips. The corresponding coverage at $\varphi_{\mathrm{Ge}-\mathrm{Nb}}=3.5 \mathrm{eV}$ was assumed to be monoatomic layer. A control experiment carried out for tungsten emitter confirmed the well-known data on work function $\left(\varphi_{\mathrm{Ge}-\mathrm{W}}=5.1 \pm 0.05 \mathrm{eV}\right.$ [5]) and on total energy distribution of field emitted electrons for Ge-W system [4, 11]. It should be noted that the volume diffusion (penetration) of Ge atoms into $\mathrm{Nb}$ emitter (which may be responsible for such an unusual dependence $\varphi(\theta)$ ) is considered as unlikely at room temperature.

During the deposition germanium is primarily observed on the (111) and (111) planes of niobium emitter (Fig. 4a). With increasing Ge coverage the contribution of the (111) and (111) planes into total emission increases (Fig. 4b). Observation of the emission patterns with increasing coverage shows a rather granular structure. Both an adsorption selectivity and a localized nucleation are observed even for submonolayer coverage $(\theta \geq 0.1)$. In the case of tungsten, cluster formation does not take place even at monoatomic (or more) layer (Fig. 4c). Thus adsorption of $\mathrm{Ge}$ on $\mathrm{W}$ is much more uniform than in the case of $\mathrm{Ge}$ on $\mathrm{Nb}$.

Adsorption activity of niobium surface in relation to residual gases $\left(\mathrm{N}_{2}, \mathrm{H}_{2}\right.$, $\mathrm{CO}, \mathrm{CO}_{2}$ ) under UHV condition was lowered after being covered with a very thin $(\theta=0.1)$ germanium film (Fig. 5). Such passivation of niobium surface as in case of tungsten [12] may be explained by localized bond formation between substrate atoms and adsorbed Ge atoms and their interaction with each other [13]. 


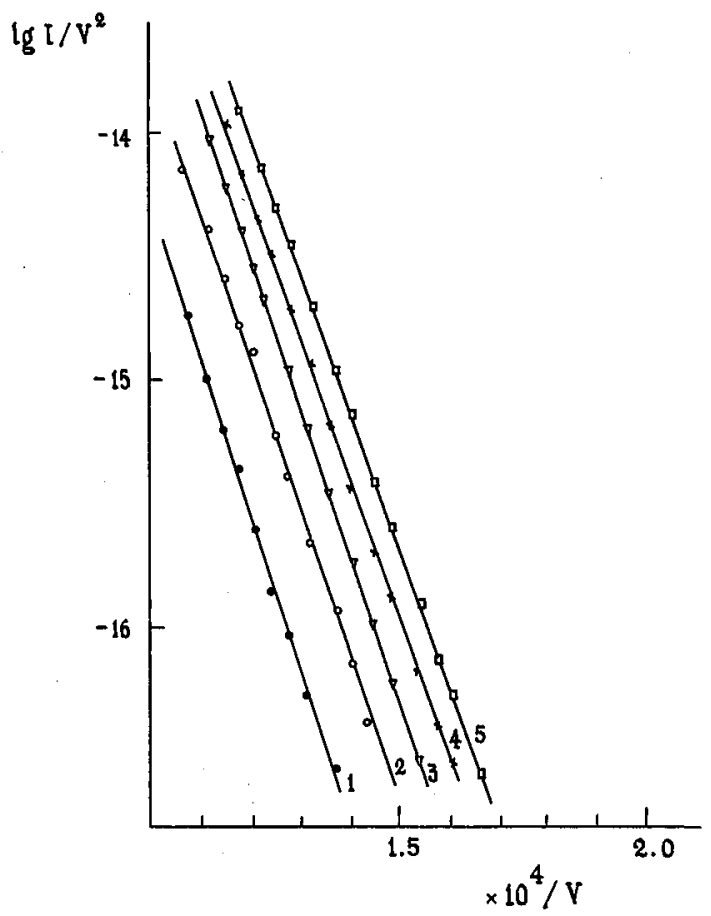

Fig. 2. Fowler-Nordheim plots of (1) clean $\mathrm{Nb}$ emitter $\left(\varphi_{\mathrm{Nb}}=4.0 \mathrm{eV}\right),(2)$ to $(5) \mathrm{Ge}$ covered Nb: (2) $\theta=0.14$, (3) $\theta=0.25$, (4) $\theta=0.40$, (5) $\theta=1.0$.

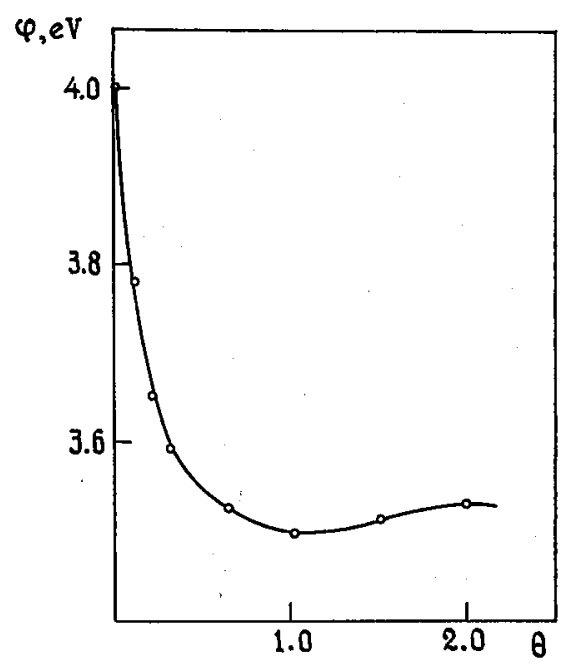

Fig. 3. Plot of the variation of work function with coverage for Ge adsorption on Nb. 

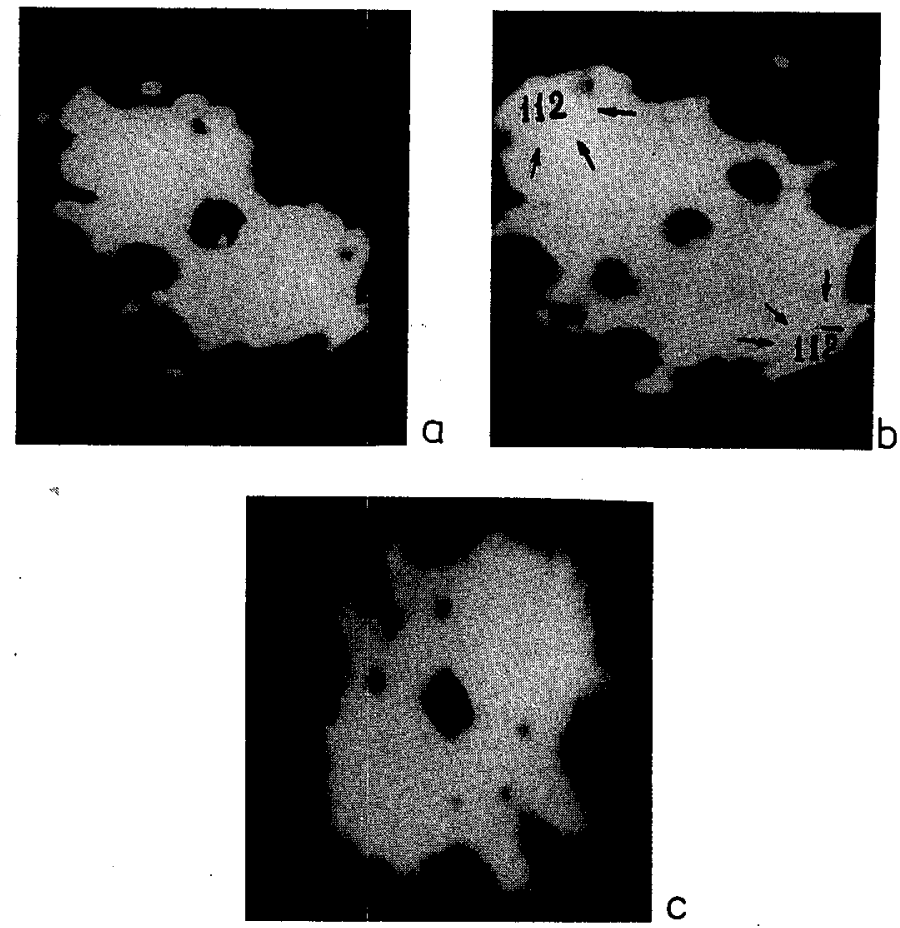

Fig. 4. FEM patterns of niobium emitter after deposition of germanium at $300 \mathrm{~K}$ : (a) $\theta=0.1$, (b) $\theta=1.5$; (c) field emission pattern of one monolayer of Ge deposited on $\mathrm{W}$ at $300 \mathrm{~K}$.

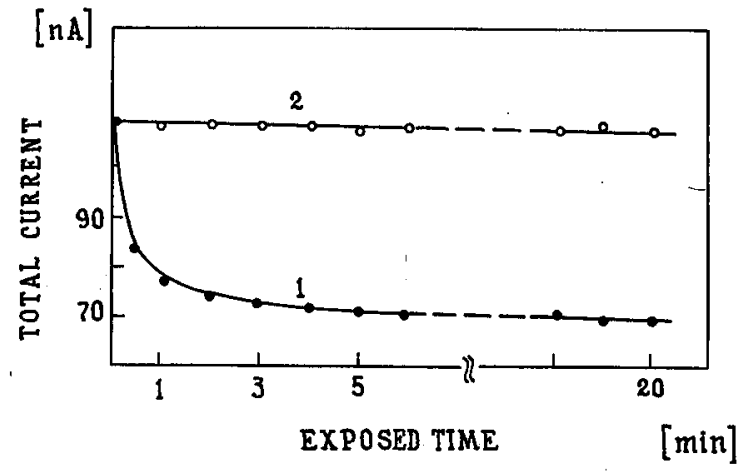

Fig. 5. Change in total field emission current with time at constant anode voltage. (1) clean $\mathrm{Nb}$; (2) Ge deposited on $\mathrm{Nb} \theta=0.1$. Residual gas pressure $10^{-8} \mathrm{~Pa}$.

\subsection{Spectral density functions}

Figure 6 shows the spectral density function $S(f)$ of the current fluctuations from the (124) plane of thermally smoothed tungsten field emitter (curve 1). It is 
clear that the $S(f)$ curve in the frequency range $1-10^{3} \mathrm{~Hz}$ is well approximated by a $1 / f^{\gamma}$ dependence, where the slope of the $S(f) \gamma=1.3$, and values of noise power are in good agreement with values obtained in [14]. It should be noted that due to

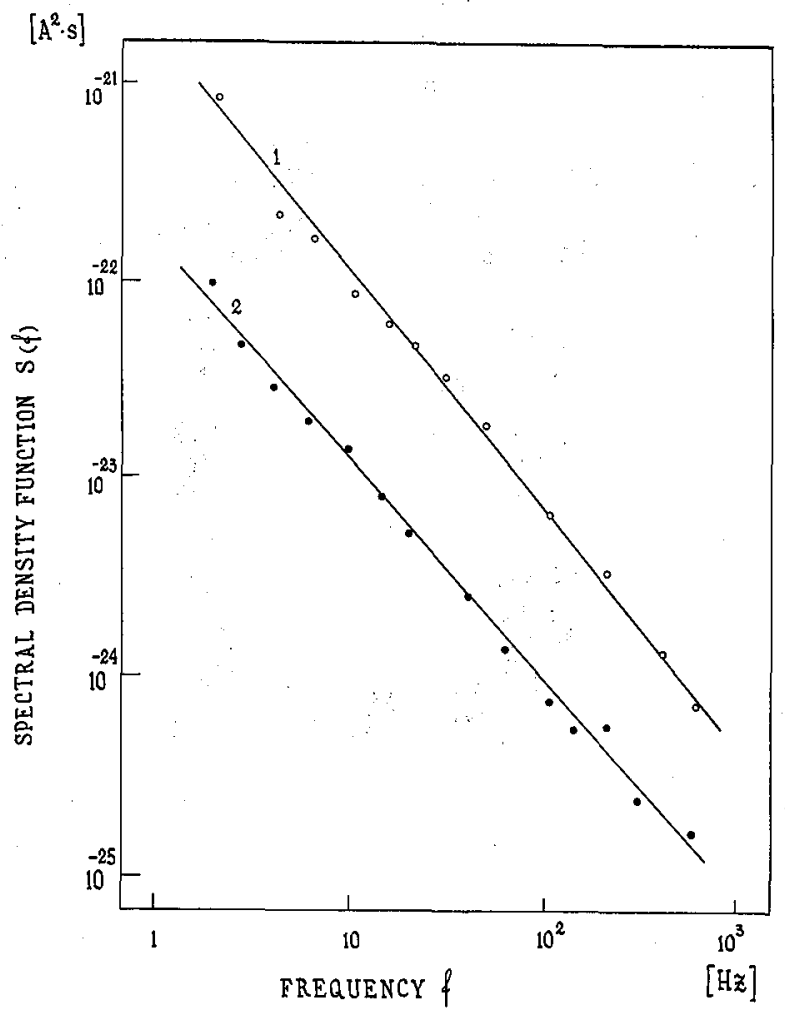

Fig. 6. Experimental spectral density functions of the field emission noise for the (124) plane of tungsten with Ge-adsorption at room temperature: (1) zero coverage, the slope is -1.3 ; (2) $\theta=1.0$, the slope is -1.0 . The probe current was $9.5 \mathrm{nA}$.

higher sensitivity of the measurement technique the flicker noise was observed in our case at $T=300 \mathrm{~K}$ also unlike the results received in [14]. Deposition of germanium monolayer results in essential lowering of noise power level and its higher stability (Fig. 6, curve 2); the slope of the spectral density function becomes equal to 1 , which is in good agreement with tungsten surface passivation phenomena [12] taking place under these conditions. The influence of germanium adsorption on field emission current noise spectra of niobium is shown in Fig. 7, in which one can observe some characteristic knee frequencies, position of which depend on $\mathrm{Ge}$ coverage and on the emitter temperature (Fig. 8). This result confirms essential contribution of mobile adatoms or clusters into the flicker noise formation. Thick layers of germanium both on $\mathrm{Nb}$ and on $\mathrm{W}$ surfaces manifest similar behaviour of noise spectra, which may be related with appearance of bulk semiconductor 


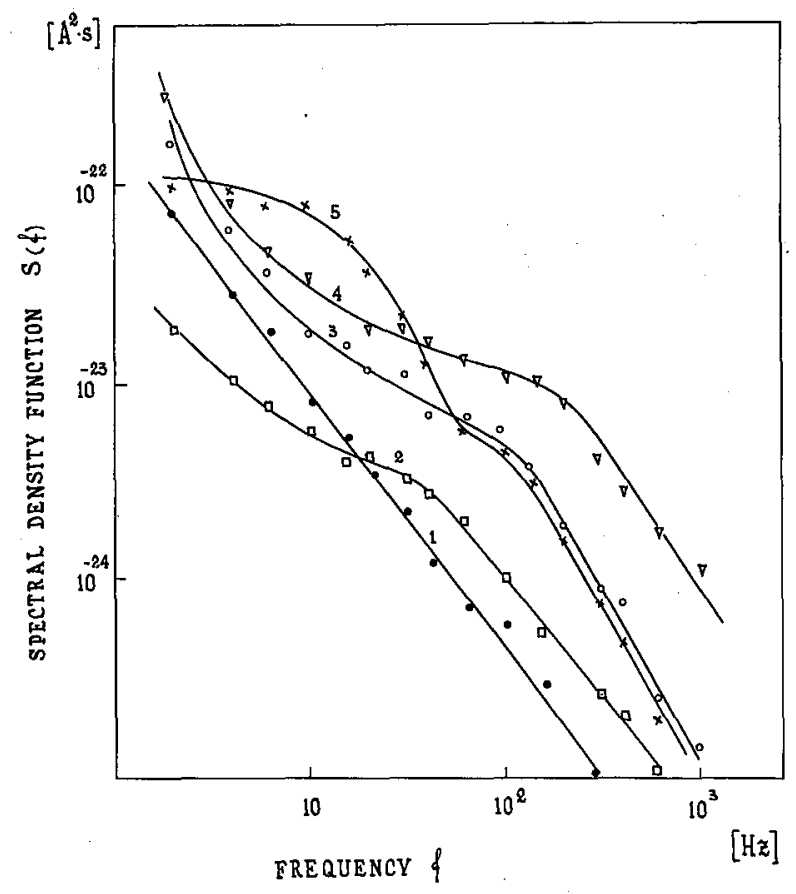

Fig. 7. Experimental spectral density functions of the (111) plane of niobium with germanium adsorption at room temperature: (1) clean $\mathrm{Nb},(2) \theta=0.14$, (3) $\theta=0.25$, (4) $\theta=0.40$, (5) $\theta=1.0$. The probe current was $9.5 \mathrm{nA}$.

properties of adlayer (Fig. 7, curve 4) [15].

\subsection{Spectral density functions dependence on temperature}

Field emission noise spectra of $\mathrm{Ge}-(111) \mathrm{Nb}$ system obtained at some characteristic temperatures and Ge coverage $\theta=0.1$ (in this case the $\mathrm{Nb}$ surface becomes passive and stable enough) are given in Fig. 8. At coverages $\theta>1.0$ and temperature $T>300 \mathrm{~K}$ on the planes $\{111\}$ of niobium emitter non-limited surface diffusion takes place, which may be observed in field emission image. For $\theta \leq 0.1$ and $T \geq 775 \mathrm{~K}$ surface diffusion in direction $\langle 111\rangle \rightarrow\langle 112\rangle$ is characterized by activation energy threshold $Q$. Characteristic knee frequencies in Fig. 8 will belong to Arrhenius plot. Corresponding value $Q$ determined from the data of Fig. 7 and Fig. 8 was found to be equal to $0.74 \pm 0.05 \mathrm{eV}$. Suppose the main mechanism of the flicker noise stems from diffusion of mobile atoms or clusters, "noise activation energy" in these conditions will be close to the activation energy for surface diffusion. Relatively low value of $Q$ confirms the presence of mobile Ge adatoms on $\mathrm{Nb}$ surface.

In principle, such a behaviour of noise spectra can be described by the Gesley-Swanson's model of diffusive equilibrium density fluctuations [16], but in 


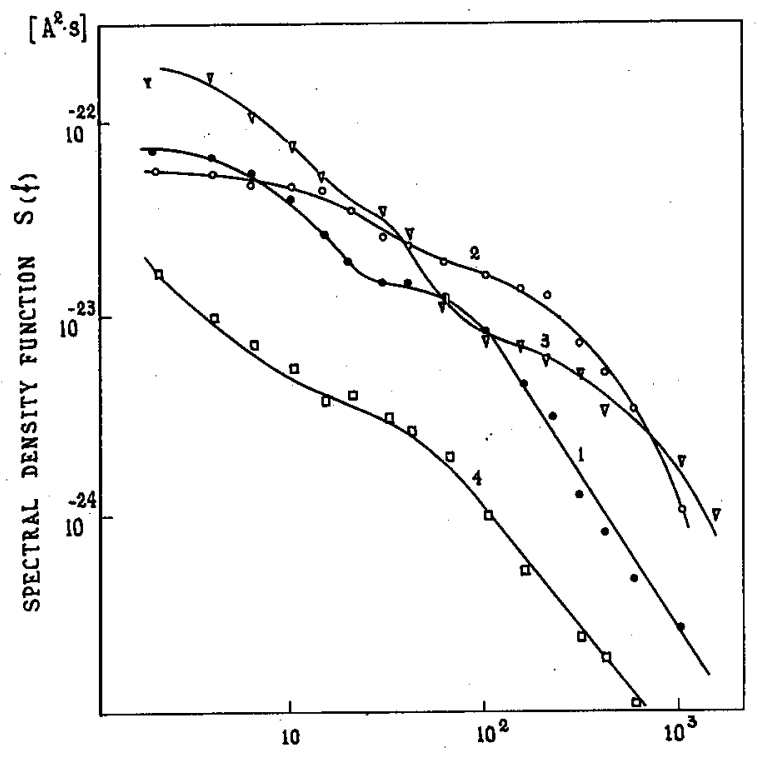

FREQUENCY \&

$[\mathrm{Hz}]$

Fig. 8. Experimental spectral density functions of the (111) plane of niobium with Ge submonolayer $(\theta=0.1)$ at different emitter temperatures: (1) $T=775 \mathrm{~K}$; (2) $T=850 \mathrm{~K}$; (3) $T=1125 \mathrm{~K}$; (4) $T=300 \mathrm{~K}$ (for comparison). The probe current was $9.5 \mathrm{nA}$.

this case the use of a simpler Kleint's model [17] seems more expedient. According to the latter the low-frequency shoulder of the $S(f)$ curve corresponds to the frequency $f_{l}=N v q$, where $N$ is the density of adsorption centres, $v$ is the velocity of diffusing (migrating) particle, $q$ is the capture cross-section of migrating particle. From $f_{l}$ and the slopes of the spectral density function for curves in Fig. 7 diffusion coefficient $D$ can be calculated using the relation $D=f_{l} / 2(2-\gamma) N$. In the range $0.1 \leq \theta \leq 1.0$ the values of $D$ were almost the same (at average $D \approx 6 \times 10^{-13}$ $\left.\mathrm{cm}^{2} / \mathrm{s}\right)$.

Visible thermodesorption of germanium from niobium surface was already observed at $T \geq 1150 \mathrm{~K}$ which indicates that the energy of adatom binding is less than in the case of tungsten and confirms the tendency to cluster formation.

\section{Summary}

1. Anomalous character of the variation of work function with coverage (in comparison with the $\mathrm{Ge}-\mathrm{W}$ system) is observed. The field emission work function for monolayer Ge film deposited on $\mathrm{Nb}$ at $300 \mathrm{~K}$ is found to be $3.5 \mathrm{eV}$.

2. Passivation of the $\mathrm{Nb}$ surface is observed after adsorption of more than 0.1 monolayer of $\mathrm{Ge}$ and is accompanied by essential reduction of field emission 
noise power; both for Ge-Nb system $(\theta=0.1)$ and for $\mathrm{Ge}-\mathrm{W}$ system $(\theta=1.0)$ the slope of the $S(f)$ is equal to 1 .

3. Threshold character of surface diffusion of Ge adatoms on $\mathrm{Nb}$ surface with activation energy $Q=0.74 \pm 0.05 \mathrm{eV}$ is observed in direction $\langle 111\rangle \rightarrow\langle 112\rangle$ for submonolayer $(\theta \leq 0.1)$ coverages.

\section{Acknowledgements}

The authors would like to thank Drs. S.S. Ghots and Yu.M. Ymaguhuzin for many stimulating discussions and to acknowledge the technical assistance of $\mathrm{Mr}$. V.M. Lobanov.

\section{References}

[1] L.A. Bolshov, A.P. Napartovich, A.G. Naumovets, Usp. Fiz. Nauk 122, 125 (1977).

[2] N. Nikolau, A. Modinos, J. Phys. C: Solid State Phys. 4, 2859 (1971).

[3] A. Kiejna, Ph. Niedermann, O. Fischer, Appl. Phys. A 30, 331 (1990).

[4] N.V. Mileslıkina, I.L. Sokolskaya, Fiz. Tverd. Tela 7, 1043 (1965).

[5] Yu.V. Zubenko, M.B. Ishmukhametov, Poverkhn. Fiz. Khim. Mekh. 8, 68 (1983).

[6] M. Drechsler, in: Surface Mobilities on Solid Materials, Ed. Yu Thien Binh, Plenum Press, New York 1983, p. 405.

[7] R. Klein, J. Chem. Phys. 21, 1177 (1953).

[8] L. Schmidt, R. Gomer, J. Chern. Phys. 42, 3573 (1967).

[9] R.Z. Bakltizin, S.S. Ghots, Prib. Teklh. Eksp. (USSR) 3, 216 (1985).

[10] S.L. Marple, Jr., Digital Spectral Analysis with Applications, Prentice-Hall, New Jersey 1987, p. 76.

[11] H.E. Clark, Ph.D. Thesis, American University, Washington, D.C., 1971.

[12] I.L. Sokolskaya, N.V. Mileshkina, Surf. Sci. 15, 109 (1969).

[13] E.Ya. Zandberg, E.V. Rut'kov, A.Yá. Tondegode, J. Tech. Phys. (Poland) 45; 1884 (1975).

[14] T. Biernat, Ch. Kleint, Acta Univ. Wratisl. 607, 13 (1981).

[15] R.Z. Bakhtizin, S.S. Ghots, J. Phys. 50-C8, 103 (1989).

[16] M.Gesley, L.W. Swanson, Phys. Rev. A 37, 4879 (1988).

[17] Ch. Kleint, Physica 68, 382 (1973). 